\title{
Surface display of an anti-DEC-205 single chain Fv fragment in Lactobacillus plantarum increases internalization and plasmid transfer to dendritic cells in vitro and in vivo
}

Michon Christophe ${ }^{1,2}$, Katarzyna Kuczkowska ${ }^{3}$, Philippe Langella ${ }^{1,2}$, Vincent G H Eijsink ${ }^{3}$, Geir Mathiesen ${ }^{3}$ and Jean-Marc Chatel ${ }^{1,2^{*}}$

\begin{abstract}
Background: Lactic acid bacteria (LAB) are promising vehicles for delivery of a variety of medicinal compounds, including antigens and cytokines. It has also been established that $L A B$ are able to deliver CDNA to host cells. To increase the efficiency of LAB-driven DNA delivery we have constructed Lactobacillus plantarum strains targeting DEC205, which is a receptor located at the surface of dendritic cells (DCs). The purpose was to increase uptake of bacterial cells, which could lead to improved cDNA delivery to immune cells.

Results: Anti-DEC-205 antibody (aDec) was displayed at the surface of L. plantarum using three different anchoring strategies: (1) covalent anchoring of aDec to the cell membrane (Lipobox domain, Lip); (2) covalent anchoring to the cell wall (LPXTG domain, CWA); (3) non-covalent anchoring to the cell wall (LysM domain, LysM). aDec was successfully expressed in all three strains, but surface location of the antibody could only be demonstrated for the two strains with cell wall anchors (CWA and LysM). Co-incubation of the engineered strains and DCs showed increased uptake when anchoring aDec using the CWA or LysM anchors. In a competition assay, free anti-DEC abolished the increased uptake, showing that the internalization is due to specific interactions between the DEC-205 receptor and aDec. To test plasmid transfer, a plasmid for expression of GFP under control of an eukaryotic promoter was transformed into the aDec expressing strains and GFP expression in DCs was indeed increased when using the strains producing cell-wall anchored aDec. Plasmid transfer to DCs in the gastro intestinal tract was also detected using a mouse model. Surprisingly, in mice the highest expression of GFP was observed for the strain in which aDec was coupled to the cell membrane.

Conclusion: The results show that surface expression of aDec leads to increased internalization of $L$. plantarum and plasmid transfer in DCs and that efficiency depends on the type of anchor used. Interestingly, in vitro data indicates that cell wall anchoring is more effective, whereas in vivo data seem to indicate that anchoring to the cell membrane is preferable. It is likely that the more embedded localization of aDec in the latter case is favorable when cells are exposed to the harsh conditions of the gastro-intestinal tract.
\end{abstract}

Keywords: Dendritic cell, DEC-205, Lactic acid bacteria, Drug delivery, Cellular targeting, Surface display

\section{Background}

DNA vaccination relies on the injection of a plasmid containing cDNA under control of an eukaryotic

\footnotetext{
*Correspondence: jean-marc.chatel@jouy.inra.fr

1 INRA, UMR1319 MICALIS, Bat 440, R-2, 78352 Jouy-en-Josas, France Full list of author information is available at the end of the article
}

promoter into host cells. It has been shown that DNA vaccination is able to elicit immune responses comparable to the responses generated by attenuated pathogens [1]. DNA vaccines can be administered by intramuscular injection of DNA, but this strategy needs trained staff and equipment, and may in some cases suffer from 
DNA instability [2]. Mucosal delivery of the vaccines is of interest because it can elicit both local and systemic immunization. Because DNA is very sensitive to the physico-chemical conditions in the gut, the use of DNA vaccination at the mucosal level implies the development of vectors able to protect the DNA. Alternatives include encapsulation of the cDNA in nanoparticles of polysaccharides or in liposomes, as well as the use of bacterial vectors [3-5].

Lactic acid bacteria (LAB) including Lactobacillus, Lactococcus, Streptococcus or Enterococcus are Gram positive bacteria. $\mathrm{LAB}$ are present in a wide range of ecological niches such as plant material and the gastro intestinal tract (GIT) and they have been used for thousands years in fermented food products. LAB have been given the GRAS (generally recognized as safe) status by the World Health Organization and several LAB strains have probiotic properties. Probiotic effects of LAB have been shown to be beneficial in relation to lactose intolerance [6], diarrhea [7], allergy [8, 9], inflammatory bowel disease (IBD) [10] and cancer [11]. Notably, the beneficial effects of LAB include immunomodulatory effects and an adjuvant-type of action [12]. One of the LAB for which such effects have been studied in most detail is Lactobacillus plantarum [12] [8] [13]. Importantly, LAB resist low $\mathrm{pH}$ and the harsh conditions in the GIT, making them a vector of choice for oral administration.

The possibility of LAB delivering cDNA to host cells has proven its efficiency in several applications. The first demonstration concerned a Lactococcus lactis strain carrying cDNA encoding $\beta$-lactoglobulin (BLG), one of the major allergens in milk $[14,15]$. Recently, it was shown that $L$. lactis carrying cDNA encoding IL-10 under control of the eukaryotic cytomegalovirus (CMV) promoter has protective effects in a mouse model of trinitrobenzene sulfonic (TNBS) acid-induced colitis [16]. Delivery of a DNA vaccine by $L$. acidophilus had a positive effect in protecting mice from foot and mouth disease [17].

Enhancing interactions between host cells and LAB may be one way to increase the transfer efficiency of cDNA-based vaccines, and therefore, several studies aimed at targeting specific cellular populations have been performed. For example, fibronectin binding protein A (FnBPA) from Staphylococcus aureus and mutated internalin A (InlA) from Listeria monocytogenes have been successfully expressed at the surface of L. lactis [18, 19]. These two proteins are responsible for binding of $S$. aureus and L. monocytogenes, respectively, to epithelial cells by interacting with specific receptors [20,21]. Binding is the first step towards internalization and it was indeed shown that transfer of a plasmid expressing either GFP or BLG was enhanced when LAB expressed FnBPA or mutated InlA $[18,19]$.
Dendritic cells (DCs) are antigen presenting cells and thus major players in the immune response. They are present in tissue mucosa, under the epithelial barrier of the gut, and they can extend dendrites through tight junctions to sample antigens in the lumen [22]. DCs are an important link between the exterior and the immune system. They have numerous different pattern recognition receptors (PRRs) and when they encounter a potential pathogen, they switch to an activated state [23]. Once activated, they migrate to lymph nodes where they stimulate $\mathrm{T}$ cells proliferation and differentiation.

One of the PRRs present in DCs is DEC-205 (or CD-205) which is a C-type lectin receptor involved in recognition of ligands expressed during apoptosis and necrosis of cells [24], in recognition of CpG oligonucleotides [25] and in antigen processing [26]. This receptor has been a target in several vaccine improvement studies [27]. For example, tumor associated antigen HER2/ neu has been fused to a single chain $\mathrm{Fv}$ fragment $(\mathrm{ScFv})$ targeting DEC-205. Administration of the fusion protein elicited a higher cellular and humoral response and had a stronger protective effect against tumor formation, compared to the antigen alone [28]. DEC-205 is also a target to induce tolerance to substances. For example, Bruder et al. [29] showed that fusing a tolerogenic vaccine against type 1 diabetes to an anti-DEC-205 antibody protected mice from developing this autoimmune disease. These effects are likely due to the fact that when an antigen is endocytosed via DEC-205, it is presented to the immune system through both MHC class I and MHC class II molecules, thus to both CD4+ and CD8+ T cells [30].

In the present study, we investigated the potential of targeting DEC-205 as a tool to enhance interactions between LAB and DCs and thus plasmid DNA delivery. We have constructed strains of L. plantarum expressing a recombinant $\mathrm{ScFv}$ against murine DEC-205 (aDec) at their surface, using three different surface anchors. We show that surface localization of aDec in L. plantarum has the potential to increase internalization of the bacterium and plasmid transfer both in cell culture and in mice and that this potential depends on the type of surface anchor.

\section{Results}

\section{Construction of recombinant Lactobacillus plantarum strains producing the anti-DEC-205 ScFv}

A DNA fragment encoding the anti-mouse Dec205 ScFv (aDec) preceded by a HA-tag for immune detection was cloned into plasmids previously developed for production and surface localization of proteins in $L$. plantarum $(32,26)$ using three different types of anchors as outlined in Figure 1a, b. The anchors are: a Lipobox membrane 


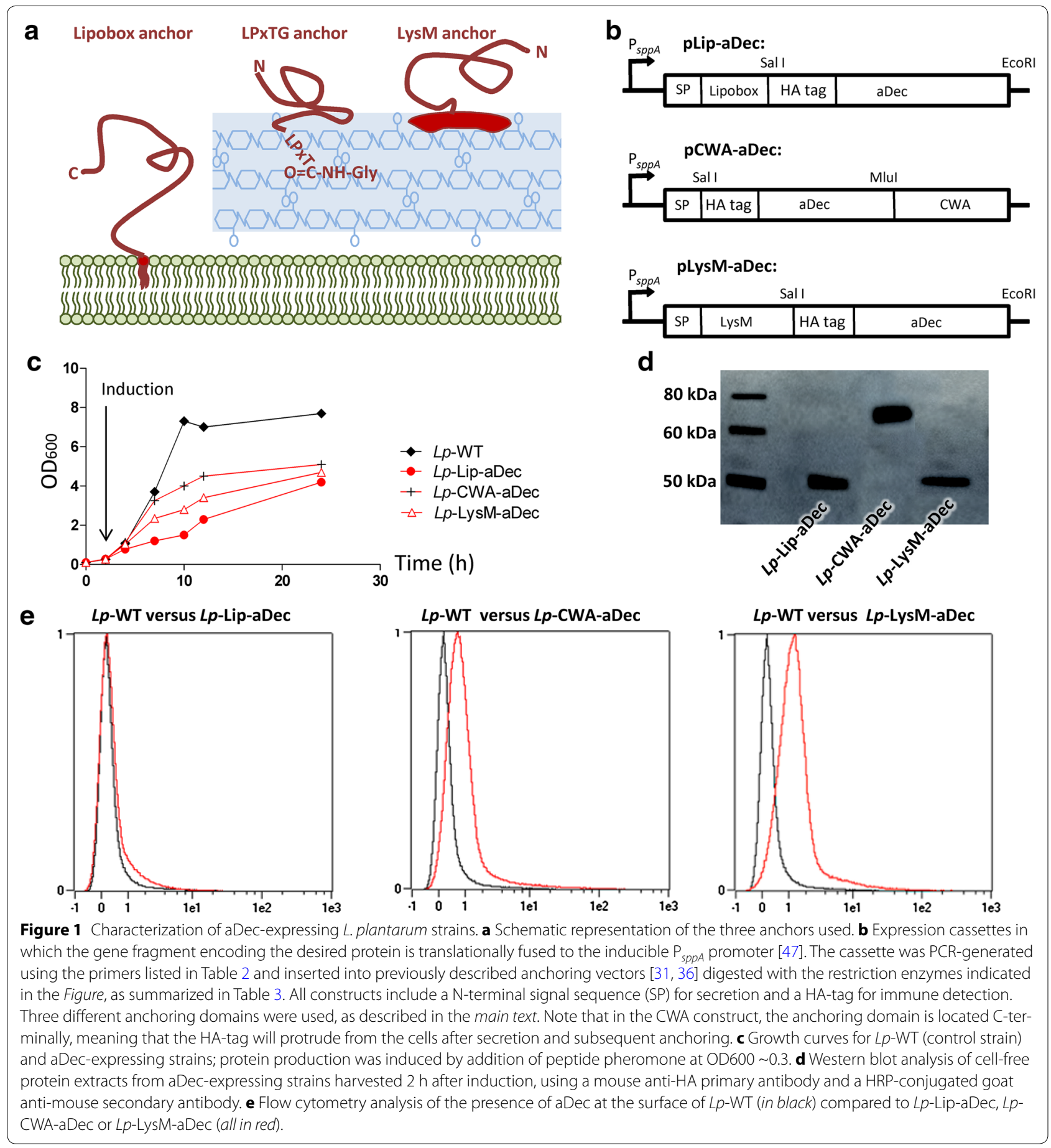

anchor for covalent coupling of aDec to a membrane component, a cell wall anchor based on sortase-catalyzed covalent coupling to the peptidoglycan and a cell wall anchor based on non-covalent interactions between a LysM domain and the cell wall. The three resulting plasmids, pLip-aDec, pCWA-aDec and pLysM-aDec (Figure $1 \mathrm{~b}$ ) were transformed into L. plantarum. In addition, we used $L p$-WT, containing a control plasmid (pEV) [31] that does not encode for the anchors or aDec, as a negative control. The growth rate of strains producing aDec was substantially lower compared to control strain (Figure 1c), in particular for the strain harboring the lipoprotein anchor. Still, all strains showed reasonable growth and bacteria harvested $2 \mathrm{~h}$ after induction, where all 
transformants have a similar $\mathrm{OD}_{600}$ (Figure 1c) and similar cell counts, were used for further studies.

Western blot analysis of protein extracts of induced strains using anti-HA antibodies revealed the presence of HA tagged proteins with correct molecular size in all three aDec-producing strains (Figure 1d). To check surface localization we labeled the strains with a fluorescent anti-HA antibody and tested antibody binding by flow cytometry. $L p$-CWA-aDec and $L p$-LysM-aDec, but not $L p$-Lip-aDec showed higher fluorescence than $L p$-WT (Figure 1e). Taken together these results show that aDec is expressed in all three aDec-producing strains. However, aDec is only detectable at the surface in the two strains where aDec is fused to the cell wall anchors.

\section{Internalization of aDec-displaying L. plantarum by dendritic cells}

To test interactions of the recombinant strains with DCs derived from humans (hDCs) or mice bone marrow dendritic cells (BMDCs), we first performed internalization assays. Figure 2 shows that the number of internalized bacteria was significantly higher after co-incubation with $L p$-CWA-aDec and $L p$-LysM-aDec compared to $L p$-WT or $L p$-Lip-aDec. To investigate the specificity of the interactions between the displayed anti-mouse $\mathrm{ScFv}$ and human DCs, a competition experiment was carried in which the hDCs were pre-treated with a monoclonal antibody against human DEC-205. The results (Figure 2a) clearly show that increased internalization caused by surface display of aDec is abolished in the presence of the competing free antibody, which proofs that increased internalization is due to a specific interaction between the recombinant strains and DEC-205 on the DCs.

\section{Effect of aDec expression on plasmid transfer to dendritic cells}

To investigate the potential of the recombinant strains for plasmid transfer we transformed the strains with an additional plasmid, pValac-GFP [32] for expression of GFP cDNA under the control of the eukaryotic CMV promoter. We thus obtained $L p$-WT/pValac-GFP, $L p$-Lip-aDec/ pValac-GFP, $L p$-CWA-aDec/pValac-GFP and $L p$-LysMaDec/pValac-GFP (Table 1). Growth of the pValac-GFP containing double transformants was similar to growth of the corresponding single transformants depicted in Figure 1c. Figure $3 \mathrm{a}$ shows that the number of $\mathrm{hDCs}$ expressing GFP was significantly higher after co-incubation with $L p$-CWAaDec/pValac-GFP or $L p$-LysM-aDec/pValac-GFP compared to $L p$-WT/pValac-GFP or $L p$-Lip-aDec/pValac-GFP. Similar results, although with lower significance, were obtained with BMDCs (Figure 3b). Competition experiments with the free anti DEC-205 antibody showed that increased plasmid transfer is due to a specific interaction between the recombinant strains and DEC-205 on the DCs.

\section{Plasmid delivery from bacteria to dendritic cells in vivo}

To investigate the potential of the recombinant strains for plasmid transfer in vivo the recombinant strains



Figure 2 Effect of surface expression of aDec on internalization of $L$. plantarum by DCs. a Monocyte derived hDCs were co-incubated with $L p$-WT, Lp-Lip-aDec, Lp-CWA-aDec or Lp-LysM-aDec with or without addition of a free anti human DEC205 antibody. After incubation, the hDCs were washed and non-internalized bacteria were killed by treatment with gentamicin. The numbers of internalized bacteria were counted as CFU. Same amount of DCs was used in each experiment. b BMDCs were co-incubated with $L p$-WT, $L p$-Lip-aDec, $L p$-CWA-aDec or $L p$-LysM-aDec. After incubation, the BMDCs were washed and non-internalized bacteria were killed by treatment with gentamicin. The numbers of internalized bacteria were counted as CFU. Each point represents independent wells and results are presented as mean \pm SEM. The results presented are from one experiment representative of three performed independently. Statistically significant differences are indicated as follows: ${ }^{*} p<0.05 ;{ }^{* *} p<0.01 ;{ }^{* * *} p<0.001$. 
Table 1 Bacterial strains and plasmids used in this study

\begin{tabular}{|c|c|c|c|}
\hline Designation & Strains & Characteristic & References \\
\hline & Lb. Plantarum WCFS1 & Host strain & {$[34]$} \\
\hline & E. coliTOP10 & Host strain & Invitrogen \\
\hline & $L p-W T$ & WCFS1 with $p E V$ & This study \\
\hline & Lp-WT/pValac-GFP & WCFS1 with $p E V$ and pValac-GFP & This study \\
\hline \multirow[t]{6}{*}{ aDec strains } & Lp-Lip-aDec & WCFS1 with pLip-aDec & This study \\
\hline & $L p-C W A-a D e c$ & WCFS1 with $\mathrm{pCWA-aDec}$ & This study \\
\hline & Lp-LysM-aDec & WCFS1 with pLysM-aDec & This study \\
\hline & Lp-Lip-aDec/pValac-GFP & WCFS1 with pLip-aDec and pValac-GFP & This study \\
\hline & Lp-CWA-aDec/pValac-GFP & WCFS1 with pCWA-aDec and pValac-GFP & This study \\
\hline & Lp-LysM-aDec/pValac-GFP & WCFS1 with pLysM-aDec and pValac-GFP & This study \\
\hline \multirow[t]{2}{*}{ Designation } & Plasmids & Characteristic & References \\
\hline & $\mathrm{pEV}$ & Control plasmid & {$[31]$} \\
\hline \multirow[t]{4}{*}{ aDec plasmids } & pLip-aDec & Plasmid expressing aDec with lipoanchor & This study \\
\hline & pCWA-aDec & Plasmid expressing aDec with LPXTG anchor & This study \\
\hline & pLysM-aDec & Plasmid expressing aDec with LysM anchor & This study \\
\hline & pValac-GFP & Plasmid expressing GFP under CMV promoter & {$[32]$} \\
\hline
\end{tabular}
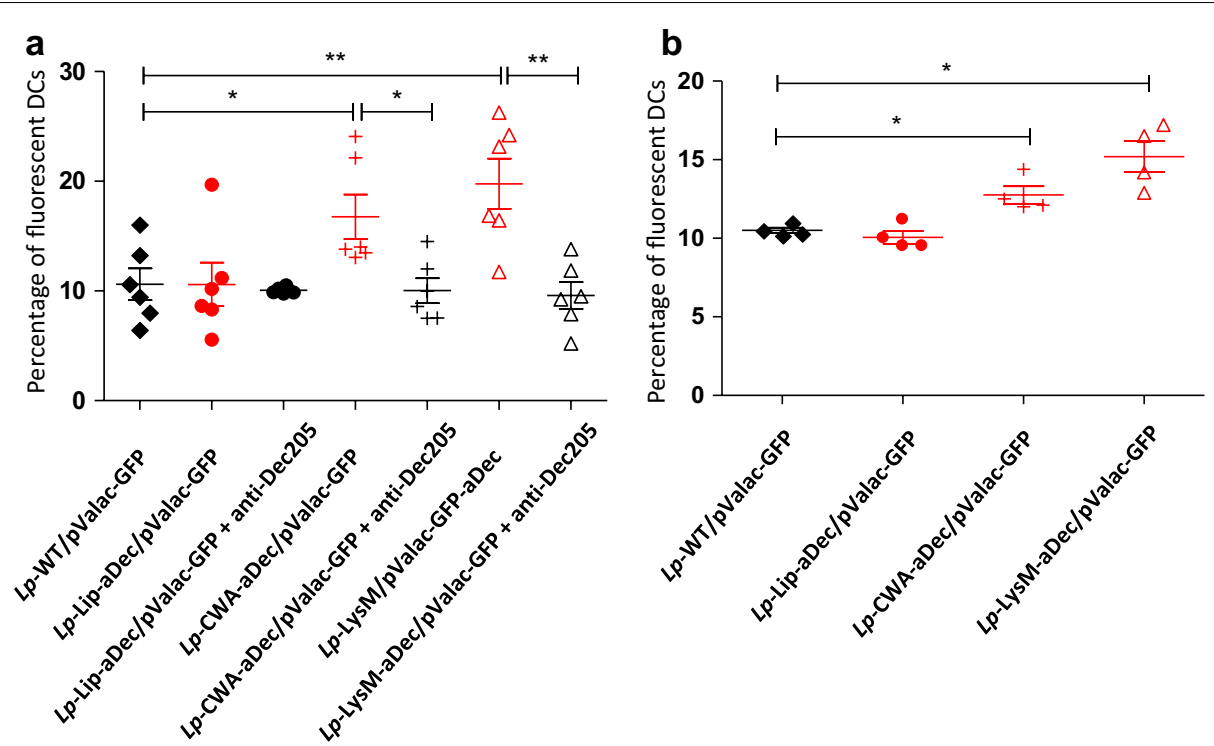

Figure 3 Effect of surface expression of aDec on plasmid delivery into DCs. a Monocyte derived hDCs were co-incubated with $L p$-WT/pValac-GFP, Lp-Lip-aDec/pValac-GFP, Lp-CWA-aDec/pValac-GFP or Lp-LysM-aDec/pValac-GFP with or without addition of a free anti-human DEC205 antibody. After incubation the percentage of green fluorescent DCs was determined by flow cytometry. b BMDCs were co-incubated with $L p$-WT/pValac-GFP, Lp-Lip-aDec/pValac-GFP, Lp-CWA-aDec/pValac-GFP or Lp-LysM-aDec/pValac-GFP. After incubation, the percentage of fluorescent DCs was determined by flow cytometry. Each point represents independent wells and the results are presented as mean $\pm \mathrm{SEM}$. The results presented are from one experiment representative of three performed independently. Statistically significant differences are indicated as follows: ${ }^{*} p<0.05 ;{ }^{* *} p<0.01$; *** $p<0.001$.

containing pValacGFP were administered to mice. After 4 days of oral administration of the four different strains, mice were euthanized and DCs were isolated from the intestine and the colon. Quantification of the fraction of fluorescent DCs by flow cytometry showed that, compared to mice fed with control strain, this fraction was higher in both the intestine (Figure 4a) and the colon (Figure $4 \mathrm{~b}$ ) for mice fed with $L p$-Lip-Dec/pValac-GFP. A similar trend, but not significant, was observed for mice fed with $L p$-LysM-aDec/pValac-GFP. Thus, expression of 

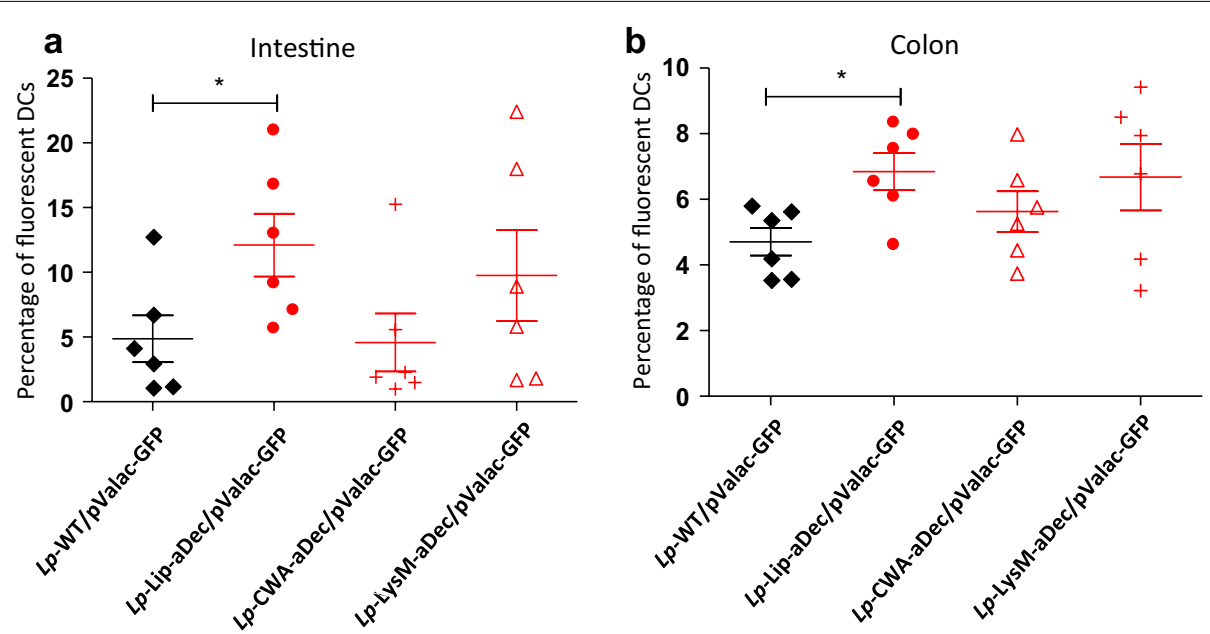

Figure 4 Effect of surface expression of aDec on plasmid transfer to mouse DCs in vivo. Mice were orally administrated with $L p-W T / p V a l a c-G F P, L p$ Lip-aDec/pValac-GFP, Lp-CWA-aDec/pValac-GFP or Lp-LysM-aDec/pValac-GFP during 4 days. At day 4 mice were sacrificed and DCs were extracted from the small intestine $(\mathbf{a})$ or the colon $(\mathbf{b})$. The percentage of DCs expressing GFP was measured by flow cytometry. Each point represents independent mice and the results are presented as mean \pm SEM. Statistically significant differences are indicated as follows: ${ }^{*} p<0.05 ;{ }^{* *} p<0.01$; ${ }^{* * *} p<0.001$.

aDec at the membrane of $L$. plantarum enhances plasmid transfer in vivo, but the preferred anchor (Lip) differs from the anchors that were most efficient in vitro (CWA and LysM).

\section{Discussion}

In the last decade promising results have been reported concerning the potential of LAB for drug delivery [33]. One of the most thoroughly studied LAB is L. plantarum whose genome was one of the first bacterial genomes to be sequenced [34]. Some L. plantarum strains have been described as probiotics [12] and the persistence of these species in the GIT is a main advantage for drug delivery. Several previous studies have shown that L. plantarum can be modified to express a variety of medicinally important proteins and that surface anchoring can be achieved in various manners $[35,36]$. Importantly, a previous study on anchoring invasin on the surface of $L$. plantarum showed that the functionality of the invasinexpressing strains depended on the type of anchor used [31]. Here, we have used these previously described anchors to explore a strategy for targeting DCs, with the ultimate goal of using this novel trait to develop more efficient LAB-based DNA vaccines.

Three surface-anchoring strategies were used to display aDec, an ScFv fragment binding to DEC-205, a C-type lectin receptor of the DCs membrane (Figure 1). Western blotting showed that the antibody fragment was indeed produced in all three corresponding strains, but in subsequent flow cytometry analyses it was only detected at the surface in two of these. The lack of detection of aDec at surface of the $L p$-Lip-aDec strain, despite the protein being highly expressed (Figure 1d), is most likely caused by the protein being embedded in the cell wall. The localization of the HA-tag at the very N-terminus of the protein, i.e. directly after the lipo-anchor, may preclude its detection with the anti-HA antibody.

In order to test the effects of our surface display of an anti-DEC205 ScFv on bacteria-DCs interactions, we used two sources of DCs, murine BMDCs and human DCs derived form monocytes which are known to express DEC205 at their surface [37] [38]. Internalization assays showed that surface display of aDec indeed increases internalization by human DCs and, to a lesser extent, murine BMDCs. Internalization by DCs was significantly higher for $L p$-CWA-aDec and $L p$-LysM-aDec strains compared to $L p$-WT and the lipoprotein construct $L p$ Lip-aDec. The lack of internalization of $L p$-Lip-aDec could be due to limited accessibility of aDec, as alluded to above. Importantly, competition assays with free antibodies confirmed that increased internalization indeed is due to specific interactions of the recombinant lactobacilli with DEC-205. This shows that L. plantarum is able to produce at its surface a fully functional recombinant antibody, which leads to increased internalization of the recombinant bacteria by the DCs.

One of the aims of the present study was to enhance the internalization efficiency of bacteria in DCs and, in addition, to optimize transfer of a functional plasmid into the DCs. To test this, we transformed the three aDec producing strains and the control strain with pValacGFP, a plasmid previously described containing an 
expression cassette with GFP cDNA under the control of an eukaryotic promoter [15]. DCs co-incubated with $L p$ CWA-aDec/pValac-GFP or $L p$-LysM-aDec/pValac-GFP showed significantly higher fractions of GFP expressing cells compared to DCs co-incubated with $L p$-Lip-aDec/ pValac-GFP or $L p$-WT-aDec/pValac-GFP. Thus, those strains in which the highest levels of surface-located aDec were detected also showed the highest levels of internalization. We have previously observed a correlation between the extent of internalization of bacteria and the extent of plasmid transfer [39]. For example expression of FnBPa or InlA at the surface of L. lactis increased internalization in Caco-2 cells and enhanced plasmid transfer both in vitro and in vivo [40-42].

To test the uptake and transfer of the GFP encoding plasmid in vivo, mice were orally administrated with relevant strains and plasmid transfer was monitored by GFP expression in DCs extracted from the GIT. Surprisingly, we observed significantly higher expression of GFP in DCs from mice administrated with $L p$-Lip-aDec/ pValac-GFP compared to other strains. The same trend was observed in mice fed with $L p$-LysM-aDec/pValacGFP, but in this case the increase was not significant. Thus, the strain that showed good protein expression but that seemed least efficient in terms of surface display and in vitro internalization and plasmid transfer gave the best in vivo results. Another factor potentially playing a role could be that the (harsh) conditions in the gut change the cell wall of the bacteria, which somehow could promote exposure of cell-wall embedded Lip-aDec.

Several studies, mainly using nanoparticles, have shown the efficiency of targeting a vaccine to DCs using aDec. For example, liposomes or bacteriophages displaying anti-DEC-205 and producing the OVA antigen induced protective effects against highly metastatic murine melanoma (B16-OVA) in mice [43, 44]. Importantly, use of DEC-205 targeting nanoparticles was shown to be more efficient than administrating nanoparticles without targeting molecules [45]. The present study adds LAB as a possible DEC-205 targeting delivery vehicle. Existing data show the possibility of replace the model GFP gene used here by other medically important genes.

\section{Conclusions}

We show that L. plantarum is able to produce and surface display a fully functional recombinant antibody, aDec, and thus target the DEC-205 receptor, which results in increased internalization of the bacteria. The results further show that the functionality of the aDecexpressing strains depends on the type of anchor used. Expression of surface-located aDec increases plasmid transfer from bacteria to DCs in vitro and in the GIT. The data indicate that to achieve efficient internalization in the GIT, a more embedded and protected localization of aDec is required.

\section{Methods}

\section{Bacterial strains and induction}

The bacterial strains and plasmids used in this study are listed in Table 1. Escherichia coli TOP10 cells (Invitrogen) were grown in brain heart infusion (BHI) broth (Oxoid) at $37^{\circ} \mathrm{C}$ with shaking. L. plantarum cells were grown statically in MRS broth (Oxoid) at $37^{\circ} \mathrm{C}$. Solid media were prepared by adding $1.5 \%(\mathrm{w} / \mathrm{v})$ agar to the broth. Plasmid constructs were first established in E. coli cells and subsequently after transformed into electrocompetent $L$. plantarum cells as described previously [46]. The antibiotic concentrations used were 5 and $200 \mu \mathrm{g} / \mathrm{mL}$ of erythromycin for L. plantarum and E. coli, respectively and $10 \mu \mathrm{g} / \mathrm{mL}$ of chloramphenicol. Induction of protein expression was done by adding the inducing peptide pheromone (SppIP) to a final concentration of $25 \mathrm{ng} / \mathrm{mL}$ at $\mathrm{OD}_{600}=0.3$, according to Sørvig et al. [47].

\section{DNA manipulations and plasmid construction}

DNA manipulations were performed essentially as previously described [48]. The primers used in this study were purchased from Operon Biotechnologies and are listed in Table 2. We used the sequence of a single chain anti mouse DEC-205 Fv fragment [49] to design a synthetic gene, codon optimized for L. plantarum expression, comprising the Fv fragment with an N-terminal HA (hemagglutinin) tag (YPYDVPDYA), which was produced by Geneart (Lifetech). The plasmid containing the synthetic gene was then used as template in subsequent PCR reactions using hot start KOD polymerase (Toyobo). Amplified PCR fragments were separated on 1\% agarose gels and purified using the NucleoSpin extract II kit (Macherey-Nagel). After purification the PCR fragments were cloned into restriction-digested plasmids containing various secretion and anchoring signals $[31,36]$ using the In-Fusion HD cloning kit (Clontech Laboratories), following the manufacturer's instructions. Vectors, primers and restriction enzymes used in cloning are summarized in Table 3 and essential aspects of the constructs

Table 2 List of PCR primers used in this study

\begin{tabular}{ll}
\hline $\begin{array}{l}\text { Primer } \\
\text { name }\end{array}$ & Sequence \\
\hline LipaDecFW & GATTGCGGCGGTCGACTATCCATATGATGTTCCAGATTATGC \\
LipaDecBW & CCGGGGTACCGAATTCTTACGATATCCCTGATGAAACTGTAAC \\
CwaaDecFW & TGCTTCATCAGTCGACTATCCATATGATGTTCCAGATTATGC \\
CwaaDecBW & GTTCAGTGACACGCGTCGATATCCCTGATGAAACTGT \\
LysMdecFW & TTGGGCCCTTGTCGACTATCCATATGATGTTCCAGATTATGC \\
LysMdecBW & CCGGGGTACCGAATTCTTACGATATCCCTGATGAAACTGTAAC \\
\hline
\end{tabular}


Table 3 Method for plasmid construction

\begin{tabular}{lllll}
\hline Plasmid name & Original plasmid & References & Restriction sites & Primer used for aDEC amplification \\
\hline pLip-aDec & pLp_1452Inv & {$[31]$} & Sall, EcoRl & LipaDecFW, LipaDecBW \\
pCWA-aDec & pLp_0373sOFAcwa2 & {$[36]$} & Sall, M/ul & CwaaDecFW, CwaaDecBW \\
pLysM-aDec & pLp_3014Inv & {$[31]$} & Sall, EcoRl & LysMdecFW, LysMdecBW \\
\hline
\end{tabular}

are displayed in Figure 1b. Plasmid DNA was purified from $E$. coli using the NucleoSpin plasmid kit (Macherey-Nagel $\mathrm{GmbH} \& \mathrm{Co}$ ). Competent L. plantarum were transformed by electroporation according to a previously described method [46]. The DNA sequences of all PCR amplicons were verified by sequence analysis.

\section{Protein extraction and western blot}

The bacteria were harvested $2 \mathrm{~h}$ after induction and washed twice with ice-cold Tris-buffered sucrose (pH 7.0, $10 \mathrm{mM} \mathrm{MgCl}$, $250 \mathrm{mM}$ sucrose). After centrifugation, the bacteria were disrupted by glass beads (106 micron, Sigma) using a FastPrep-24 instrument (MP Biomedicals) to obtain protein extracts. After cell disruption and centrifugation, cell-free supernatants were mixed with SDS-PAGE sample buffer. After boiling the samples for $7 \mathrm{~min}$, they were applied to $10 \%$ Stain-Free gels (Biorad), followed by electrophoresis with subsequent immunoblotting using the iBlot system (Thermo Fisher Scientific) according to the instructions of the manufacturer. Protein detection was performed with the SNAP i.d. System (Millipore), using a murine anti-HA antibody (Sigma) and a horseradish peroxidase (HRP)-conjugated goat anti-mouse antibody (BioRad). Proteins were visualized using the SuperSignal West Pico chemiluminescent substrate (Pierce).

\section{Detection of aDec at the surface of $L$. plantarum}

Approximately $5 \times 10^{8}$ bacterial cells determined by colony forming unit were harvested by centrifugation and washed three times with cold PBS. The bacteria were resuspended in $300 \mu \mathrm{L}$ PBS containing $1 \%$ bovine serum albumin (PBS-B) and $10 \mu \mathrm{L}$ of monoclonal Anti-HAFITC antibody $(1 \mathrm{mg} / \mathrm{mL})$. After incubation at $4^{\circ} \mathrm{C}$ for 30-60 min, the bacteria were centrifuged at $7,000 \times g$ for $2 \mathrm{~min}$ at $4{ }^{\circ} \mathrm{C}$ and washed three times with $500 \mu \mathrm{L}$ ice-cold PBS. The bacteria were subsequently fixed in $100 \mu \mathrm{L}$ of $2 \%$ paraformaldehyde (VWR) in water for 30-60 min at $4^{\circ} \mathrm{C}$. Bacteria were collected by centrifugation at $7,000 \times g$ for $2 \mathrm{~min}$, washed three times with $500 \mu \mathrm{L}$ PBS-B and resuspended in PBS. Fluorescent staining of resuspended bacteria was analyzed by flow cytometry using a MACSQuant analyzer (Miltenyi Biotec GmbH, Bergisch Gladbach, Germany), following the manufacturer's instructions.

\section{Preparation of human dendritic cells ( $\mathrm{hDCs}$ ) and FACS analysis}

Human peripheral leukocytes were prepared from blood provided by healthy volunteers according to institutional guidelines (Østfold Hospital Trust). Blood of four different donors was used. Blood was mixed with an equivalent volume of sterile PBS and half a volume of Lymphoprep (Stemcell technologies), and centrifuged for $25 \mathrm{~min}$ at $1,500 \times g$. Lymphocyte obtained with the gradient were extracted and washed three times with PBS with $10 \mathrm{~min}$ of centrifugation at $1,000 \times g$ after each step. The last centrifuge was performed at $700 \times g$ to withdraw plates. The purified peripheral leukocytes were stored in 50\% RPMI 1640 medium (PAA Laboratories) and 50\% dimethylsulfoxide (Sigma) in liquid nitrogen.

Human peripheral leukocytes were sorted using a Macs separator and Macs CD14 plus microbeads (Miltenyi Biotec) according to the supplier's instructions. CD14+ cells were grown for 6 days in RPMI 1640 medium (PAA Laboratories $\mathrm{GmbH}$ ) supplemented with $1 \mathrm{mM}$ sodium pyruvate, $50 \mathrm{mM}$ thioglycerol, $25 \mu \mathrm{g} / \mathrm{mL}$ gentamicin (Garamycin), 10\% (m/v) fetal calf serum (Gibco Life Technologies), $25 \mu \mathrm{g} / \mathrm{mL}$ of IL-4 (R\&D) and $50 \mathrm{ng} / \mathrm{mL}$ of human granulocyte macrophage colony-stimulating factor (GMCSF) (Abcam). Cells were grown in a humidified incubator at $37^{\circ} \mathrm{C}$ and $5 \% \mathrm{CO}_{2}$ for 6 days.

Number and size of hDCs cells were evaluated by incubation with mouse anti-human CD86 Alexa Fluor 700conjugated antibody (BD Biosciences) for $1 \mathrm{~h}$ at room temperature protected from light. After incubation cells were washed twice with PBS and analyzed by flow cytometry using a MACSQuant analyzer (Miltenyi Biotec $\mathrm{GmbH}$ ), following the manufacturer's instructions. Proportion above $90 \%$ of CD86 positive cells were routinely obtained.

\section{Preparation of bone marrow dendritic cells (BMDCs) and FACS analysis}

Bone marrow cells from 6 weeks Balb/c female mice (Janvier) were extracted from leg bones with a $25 \mathrm{G} \times 5 / 8$ needle (Terumo). Red blood cells were eliminated by treatment with red blood cell lysis buffer (Sigma) at room temperature, following the manufacturer's protocol. After centrifugation, the cells were washed three times with RPMI1640 (PAA Laboratories). The cells were then resuspended and grown for 10 days in RPMI 1640 
medium containing $1 \mathrm{mM}$ sodium pyruvate, $50 \mathrm{mM}$ thioglycerol, $25 \mu \mathrm{g} / \mathrm{mL}$ penicillin streptomycin (Garamycin), $10 \%$ fetal calf serum (Gibco Life Technologies), and $50 \mathrm{ng} / \mathrm{mL}$ mouse GMCSF (Biolegend), in a humidified incubator at $37^{\circ} \mathrm{C}$ with $5 \% \mathrm{CO}_{2}$.

Number and size of BMDCs cells were evaluated by incubation with alexa-fluor-488 anti-CD86 (Biolegend) and R-PE conjugated Monoclonal antibody specific to Mouse CD11c (invitrogen). Marking was analyzed by flow cytometry using a MACSQuant analyzer (Miltenyi Biotec), following the manufacturer's instructions. Proportion above $90 \%$ of CD86 and CD11c positive cells were routinely obtained.

\section{Internalization assay}

$1 \times 10^{8}$ bacteria determined by colony forming unit suspended in $100 \mu \mathrm{L} 1 \mathrm{M}$ Tris buffer, $\mathrm{pH} 8.5$, were coincubated with $1 \times 10^{5}$ BMDCs or hDCs (multiplicity of infection $=200$ ) for $2 \mathrm{~h}$ in $500 \mu \mathrm{L}$ RPMI 1640 medium without antibiotics, in $5 \% \mathrm{CO}_{2}$ at $37^{\circ} \mathrm{C}$. After incubation, the cells were washed three times with $1 \mathrm{~mL}$ PBS and incubated in $500 \mu \mathrm{L}$ RPMI 1640 containing $150 \mu \mathrm{g} /$ $\mathrm{mL}$ of gentamicin for $2 \mathrm{~h}$ at $37^{\circ} \mathrm{C}$ with $5 \% \mathrm{CO}_{2}$, in order to kill non-internalized bacteria. Subsequently, DCs were lysed in $100 \mu \mathrm{L}$ PBS with $2 \%$ Triton X-100 (Sigma). The lysates, containing internalized bacteria, were plated on MRS plates and colony forming units (CFU) were counted. Experiments were repeated three times, each time on three different mice for BMDCs. For hDCs, were repeated also three times the experiment but on the same four different blood donors.

In competition experiments, the hDCs were pre-incubated for $1 \mathrm{~h}$ with monoclonal anti-human CD205 Antibody [Purified anti-human CD205 (DEC-205) Antibody; Biolegend] at a final concentration of $2 \mu \mathrm{g} / \mathrm{mL}$, prior to addition of the bacteria.

\section{Plasmid delivery to DCs}

BMDCs or hDCs were co-incubated with the different bacterial strains as described above. After incubation, the cells were washed three times with RPMI and grown for $36 \mathrm{~h}$ in RPMI 1640 medium (PAA Laboratories $\mathrm{GmbH}$ ) containing $1 \mathrm{mM}$ sodium pyruvate, $50 \mathrm{mM}$ thioglycerol, $25 \mu \mathrm{g} / \mathrm{mL}$ penicillin streptomycin (Garamycin), 10\% fetal calf serum (Gibco Life Technologies). Thereafter approximately $1 \times 10^{6}$ harvested cells were washed three times with cold PBS and them resuspended in $300 \mu \mathrm{L}$ PBS-B. GFP expression was analyzed by flow cytometry using a MACSQuant analyzer (Miltenyi Biotec $\mathrm{GmbH}$ ), following the manufacturer's instructions. Experiments were repeated three times, each time on three different mice for BMDCs. For hDCs, were repeated also three times the experiment but on the same four different blood donors.

\section{Experimental protocol for animal experiments}

Groups of 6 C57BL/6 mice (Janvier) were fed by gavage during 4 days with $1 \times 10^{9}$ bacteria/day in $100 \mu \mathrm{L}$ PBS. At day 4 , the mice were euthanized by cervical elongation, the intestine and colon were withdrawn, opened longitudinally, and cut into $5 \mathrm{~mm}$ pieces which were washed 4 times by incubation for $20 \mathrm{~min}$ at $37^{\circ} \mathrm{C}$ in $5 \mathrm{~mL}$ PBS containing $2 \mathrm{mM}$ EDTA, with strong shaking (>250 rpm). After each incubation, the samples were centrifuged at $500 \times g$ for $5 \mathrm{~min}$ and the supernatant, containing enterocytes, was discarded. Then, the fragments were incubated three times for $45 \mathrm{~min}$ at $37^{\circ} \mathrm{C}$ with strong shaking (>250 rpm) in $100 \mathrm{U} / \mathrm{mL}$ of collagenase D (Roche), $30 \mu \mathrm{g}$ DNase 1 (Sigma), 20\% v/v fetal calf serum (Gibco Life Technologies) in PBS. The samples were centrifuged at $500 \times g$ for $5 \mathrm{~min}$ after each incubation and supernatants were collected, pooled and filtered with a $70 \mu \mathrm{m}$ Falcon cell strainer (Falcon). The extracted cells were sorted with a Macs separator using mouse CD11c beads (Miltenyi Biotec) to collect DCs, according to the manufacturer's instructions. Marking of the mouse DCs thus isolated was analyzed by flow cytometry using a MACSQuant analyzer (Miltenyi Biotec $\mathrm{GmbH})$, following the manufacturer's instructions.

\section{Statistical tests}

Statistical significance was tested using the Mann-Whitney test on Prism (GraphPad software). Results are presented as mean \pm SEM. Statistical significance was considered for $\mathrm{p}<0.05 .{ }^{*}$ is $\mathrm{p}<0.05,{ }^{* *}$ is $\mathrm{p}<0.01$ and ${ }^{* * *}$ is $\mathrm{p}<0.001$.

\section{Abbreviations}

aDec: anti-Dec-205 recombinant antibody; BLG: $\beta$-lactoglobulin; BMDC: bone marrow dendritic cell; CFU: colony forming units; CWA: cell wall anchor; DC: dendritic cell; FnBPA: fibronectin binding protein A; GIT: gastro-intestinal tract; HA: human influenza hemagglutinin; hDC: human dendritic cell; IBD: inflammatory bowel disease; InIA: internalin A; LAB: lactic acid bacteria; Lip: lipobox anchor; LysM: LysM anchor; PRR: pattern recognition receptor; ScFv: single chain Fv fragment.

\section{Authors' contributions}

$\mathrm{CM}$ and KK performed all the laboratory experiments and drafted the paper. GM, VE and PL defined the research theme, helped to orient the work and revised the manuscript. JMC designed the project, coordinated it, wrote and revised the manuscript. All authors read and approved the final manuscript.

\section{Author details}

${ }^{1}$ INRA, UMR1319 MICALIS, Bat 440, R-2, 78352 Jouy-en-Josas, France.

2 AgroParisTech, UMR MICALIS, 78352 Jouy-en-Josas, France. ${ }^{3}$ Department of Chemistry, Biotechnology and Food Science, Norwegian University of Life Sciences, Aas, Norway.

\section{Acknowledgements}

This work was funded by the Norwegian Research Council through Grant 234502, an Yggdrasil Norwegian mobility (Grant Number 227257) and a mobility grant from AgroParisTech.

Compliance with ethical guidelines

Competing interests

The authors declare that they have no competing interests. 
Received: 23 March 2015 Accepted: 24 June 2015 Published online: 04 July 2015

\section{References}

1. Glenting J, Wessels S (2005) Ensuring safety of DNA vaccines. Microb Cell Fact 4:26

2. Lechardeur D, Sohn KJ, Haardt M, Joshi PB, Monck M, Graham RW et al (1999) Metabolic instability of plasmid DNA in the cytosol: a potential barrier to gene transfer. Gene Ther 6:482-497

3. Wong BS, Yoong SL, Jagusiak A, PanczykT, Ho HK, Ang WH et al (2013) Carbon nanotubes for delivery of small molecule drugs. Adv Drug Deliv Rev 65:1964-2015

4. Yang Y, Wang S, Wang Y, Wang X, Wang Q, Chen M (2014) Advances in self-assembled chitosan nanomaterials for drug delivery. Biotechnol Adv 32:1301-1316

5. Pichon C, Billiet L, Midoux P (2010) Chemical vectors for gene delivery: uptake and intracellular trafficking. Curr Opin Biotechnol 21:640-645

6. Panel E, Nda A, Golly I (2011) Scientific opinion on the substantiation of health claims related to live yoghurt cultures and improved lactose digestion (ID 1143, 2976) pursuant to Article 13 (1) of Regulation (EC) No 1924/2006 1. EFSA J 8:1-18

7. Sazawal S, Hiremath G, Dhingra U, Malik P, Deb S, Black RE (2006) Efficacy of probiotics in prevention of acute diarrhoea: a meta-analysis of masked, randomised, placebo-controlled trials. Lancet Infect Dis 6:374-382

8. Rigaux P, Daniel C, Hisbergues M, Muraille E, Hols P, Pot B et al (2009) Immunomodulatory properties of Lactobacillus plantarum and its use as a recombinant vaccine against mite allergy. Allergy 64:406-414

9. Van der Aa LB, Heymans HS, van Aalderen WM, Sillevis Smitt JH, Knol J, Ben Amor K et al (2010) Effect of a new synbiotic mixture on atopic dermatitis in infants: a randomized-controlled trial. Clin Exp Allergy 40:795-804

10. Pagnini C, Saeed R, Bamias G, Arseneau KO, Pizarro TT, Cominelli F (2010) Probiotics promote gut health through stimulation of epithelial innate immunity. Proc Natl Acad Sci USA 107:454-459

11. Ma EL, Choi YJ, Choi J, Pothoulakis C, Rhee SH, Im E (2010) The anticancer effect of probiotic Bacillus polyfermenticus on human colon cancer cells is mediated through ErbB2 and ErbB3 inhibition. Int J Cancer 127:780-790

12. Bron PA, van Baarlen $P$, Kleerebezem M (2012) Emerging molecular insights into the interaction between probiotics and the host intestinal mucosa. Nat Rev Microbiol 10:66-78

13. Van Baarlen P, Troost FJ, van Hemert S, van der Meer C, de Vos WM, de Groot PJ et al (2009) Differential NF-kappaB pathways induction by Lactobacillus plantarum in the duodenum of healthy humans correlating with immune tolerance. Proc Natl Acad Sci USA 106:2371-2376

14. Chatel J-M, Pothelune L, Ah-Leung S, Corthier G, Wal J-M, Langella P (2008) In vivo transfer of plasmid from food-grade transiting Lactococci to murine epithelial cells. Gene Ther 15:1184-1190

15. Guimarães VD, Innocentin S, Lefèvre F, Azevedo V, Wal J-M, Langella P et a (2006) Use of native Lactococci as vehicles for delivery of DNA into mammalian epithelial cells. Appl Environ Microbiol 72:7091-7097

16. Zurita-Turk M, Del Carmen S, Santos ACG, Pereira VB, Cara DC, Leclercq SY et al (2014) Lactococcus lactis carrying the pValac DNA expression vector coding for $\mathrm{IL}-10$ reduces inflammation in a murine model of experimental colitis. BMC Biotechnol 14:73

17. Li Y-G, Tian F-L, Gao F-S, Tang X-S, Xia C (2007) Immune responses generated by Lactobacillus as a carrier in DNA immunization against foot-andmouth disease virus. Vaccine 25:902-911

18. De Azevedo M, Karczewski J, Lefévre F, Azevedo V, Miyoshi A, Wells JM et al (2012) In vitro and in vivo characterization of DNA delivery using recombinant Lactococcus lactis expressing a mutated form of L. monocytogenes internalin A. BMC Microbiol 12:299

19. Pontes D, Innocentin S, Del Carmen S, Almeida JF, Leblanc J-G, de Moreno de Leblanc A et al (2012) Production of fibronectin binding protein A at the surface of Lactococcus lactis increases plasmid transfer in vitro and in vivo. PLoS One 7:e44892

20. Mengaud J, Ohayon H, Gounon P, Mège R-M, Cossart P (1996) E-cadherin is the receptor for internalin, a surface protein required for entry of $L$. monocytogenes into epithelial cells. Cell 84:923-932
21. Sinha B, Francois P, Que Y-A, Hussain M, Heilmann C, Moreillon P et al (2000) Heterologously expressed Staphylococcus aureus fibronectinbinding proteins are sufficient for invasion of host cells. Infect Immun 68:6871-6878

22. Chieppa M, Rescigno M, Huang AYC, Germain RN (2006) Dynamic imaging of dendritic cell extension into the small bowel lumen in response to epithelial cell TLR engagement. J Exp Med 203:2841-2852

23. Kastenmüller W, Kastenmüller K, Kurts C, Seder RA (2014) Dendritic celltargeted vaccines-hope or hype? Nat Rev Immunol 14:705-711

24. Shrimpton RE, Butler M, Morel A-S, Eren E, Hue SS, Ritter MA (2009) CD205 (DEC-205): a recognition receptor for apoptotic and necrotic self. Mol Immunol 46:1229-1239

25. Lahoud MH, Ahmet F, Zhang JG, Meuter S, Policheni AN, Kitsoulis S et al (2012) DEC-205 is a cell surface receptor for CpG oligonucleotides. Proc Natl Acad Sci 109(40):16270-16275

26. Jiang W, Swiggard WJ, Heufler C, Peng M, Mirza A, Steinman RM et al (1995) The receptor DEC-205 expressed by dendritic cells and thymic epithelial cells is involved in antigen processing. Nature 375:151-155

27. Sehgal K, Dhodapkar KM, Dhodapkar MV (2014) Targeting human dendritic cells in situ to improve vaccines. Immunol Lett 162:59-67

28. Wang B, Zaidi N, He L-Z, Zhang L, Kuroiwa JM, Keler T et al (2012) Targeting of the non-mutated tumor antigen HER2/neu to mature dendritic cells induces an integrated immune response that protects against breast cancer in mice. Breast Cancer Res 14:R39

29. Bruder D, Westendorf AM, Hansen W, Prettin S, Gruber AD, Qian $Y$ et al (2005) On the edge of autoimmunity: T-cell stimulation by steady-state dendritic cells prevents autoimmune diabetes. Diabetes 54(December):3395-3401

30. Mahnke K, Guo M, Lee S, Sepulveda H, Swain SL, Nussenzweig M et al (2000) The dendritic cell receptor for endocytosis, DEC-205, can recycle and enhance antigen presentation via major histocompatibility complex class II-positive lysosomal compartments. J Cell Biol 151:673-684

31. Fredriksen L, Kleiveland CR, Olsen Hult LT, Lea T, Nygaard CS, Eijsink VG et al (2012) Surface display of N-terminally anchored invasin by Lactobacillus plantarum activates NF-kB in monocytes. Appl Environ Microbiol 78:5864-5871

32. Guimaraes V, Innocentin S, Chatel J-M, Lefevre F, Langella P, Azevedo V et al (2009) A new plasmid vector for DNA delivery using Lactococci. Genet Vaccines Ther 7:4

33. Wells JM, Mercenier A (2008) Mucosal delivery of therapeutic and prophylactic molecules using lactic acid bacteria. Nat Rev Microbiol 6:349-362

34. Kleerebezem M, Boekhorst J, van Kranenburg R, Molenaar D, Kuipers OP, Leer R et al (2003) Complete genome sequence of Lactobacillus plantarum WCFS1. Proc Natl Acad Sci USA 100(4):1990-1995

35. Cortes-Perez NG, Lefèvre F, Corthier G, Adel-Patient K, Langella P, Bermúdez-Humarán LG (2007) Influence of the route of immunization and the nature of the bacterial vector on immunogenicity of mucosal vaccines based on lactic acid bacteria. Vaccine 25:6581-6588

36. Fredriksen L, Mathiesen G, Sioud M, Eijsink VGH (2010) Cell wall anchoring of the 37-Kilodalton oncofetal antigen by Lactobacillus plantarum for mucosal cancer vaccine delivery. Appl Environ Microbiol 76:7359-7362

37. Inaba K, Swiggard WJ, Inaba M, Meltzer J, Mirza A, Sasagawa T et al (1995) Tissue distribution of the DEC-205 protein that is detected by the monoclonal antibody NLDC-145. I. Expression on dendritic cells and other subsets of mouse leukocytes. Cell Immunol 163:148-156

38. Guo M, Gong S, Maric S, Misulovin Z, Pack M, Mahnke Ket al (2000) A monoclonal antibody to the DEC-205 endocytosis receptor on human dendritic cells. Hum Immunol 61:729-738

39. Innocentin S, Guimarães V, Miyoshi A, Azevedo V, Langella P, Chatel J-M et al (2009) Lactococcus lactis expressing either Staphylococcus aureus fibronectin-binding protein A or Listeria monocytogenes internalin A can efficiently internalize and deliver DNA in human epithelial cells. Appl Environ Microbiol 75:4870-4878

40. Anderson J, Edney R, Whelan K (2012) Systematic reviews: faecal microbiota transplantation in the management of inflammatory bowel disease. Aliment Pharmacol Ther 36:503-516

41. Pontes D, Azevedo M, Innocentin S, Blugeon S, Lefévre F, Azevedo V et a (2014) Immune response elicited by DNA vaccination using Lactococcus lactis is modified by the production of surface exposed pathogenic protein. PLoS One 9:e84509 
42. Pontes DS, de Azevedo MSP, Chatel J-M, Langella P, Azevedo V, Miyoshi A (2011) Lactococcus lactis as a live vector: heterologous protein production and DNA delivery systems. Protein Expr Purif 79:165-175

43. Van Broekhoven CL, Parish CR, Demangel C, Britton WJ, Altin JG (2004) Targeting dendritic cells with antigen-containing liposomes: a highly effective procedure for induction of antitumor immunity and for tumor immunotherapy. Cancer Res 64:4357-4365

44. Sartorius R, Bettua C, D'Apice L, Caivano A, Trovato M, Russo D et al (2011) Vaccination with filamentous bacteriophages targeting DEC-205 induces DC maturation and potent anti-tumor T-cell responses in the absence of adjuvants. Eur J Immunol 41:2573-2584

45. Cruz LJ, Rosalia RA, Kleinovink JW, Rueda F, Löwik CWGM, Ossendorp F (2014) Targeting nanoparticles to CD40, DEC-205 or CD11c molecules on dendritic cells for efficient CD8(+) T cell response: a comparative study. J Control Release 192:209-218
46. Aukrust T, Blom H (1992) Transformation of Lactobacillus strains used in meat and vegetable fermentations. Food Res Int 25:253-261

47. Sørvig E, Mathiesen G, Naterstad K, Eijsink VGH, Axelsson L (2005) Highlevel, inducible gene expression in Lactobacillus sakei and Lactobacillus plantarum using versatile expression vectors. Microbiology 151(Pt 7):2439-2449

48. Sambrook J, Fritsch E, Maniatis T (1989) Molecular cloning: a laboratory manual, 2nd edn. Cold Spring Harb Lab, Cold Spring Harbor

49. Nchinda G, Kuroiwa J, Oks M, Trumpfheller C, Chae GP, Huang Y et al (2008) The efficacy of DNA vaccination is enhanced in mice by targeting the encoded protein to dendritic cells. J Clin Invest 118:1427-1436

\section{Submit your next manuscript to BioMed Central and take full advantage of:}

- Convenient online submission

- Thorough peer review

- No space constraints or color figure charges

- Immediate publication on acceptance

- Inclusion in PubMed, CAS, Scopus and Google Scholar

- Research which is freely available for redistribution

Submit your manuscript at

www.biomedcentral.com/submit

C Biomed Central 